University of Nebraska - Lincoln

DigitalCommons@University of Nebraska - Lincoln

Andrzej Rajca Publications

Published Research - Department of Chemistry

November 2001

\title{
Magnetic Ordering in an Organic Polymer
}

\author{
Andrzej Rajca \\ University of Nebraska - Lincoln, arajca1@unl.edu \\ Jirawat Wongsriratanakul \\ Suchada Rajca \\ University of Nebraska-Lincoln, srajca1@unl.edu
}

Follow this and additional works at: https://digitalcommons.unl.edu/chemistryrajca

Part of the Chemistry Commons

Rajca, Andrzej; Wongsriratanakul, Jirawat; and Rajca, Suchada, "Magnetic Ordering in an Organic Polymer" (2001). Andrzej Rajca Publications. 4.

https://digitalcommons.unl.edu/chemistryrajca/4

This Article is brought to you for free and open access by the Published Research - Department of Chemistry at DigitalCommons@University of Nebraska - Lincoln. It has been accepted for inclusion in Andrzej Rajca Publications by an authorized administrator of DigitalCommons@University of Nebraska - Lincoln. 


\title{
Magnetic Ordering in an Organic Polymer
}

\section{Andrzej Rajca,* Jirawat Wongsriratanakul, Suchada Rajca}

\begin{abstract}
We describe preparation and magnetic properties of an organic $\pi$-conjugated polymer with very large magnetic moment and magnetic order at low temperatures. The polymer is designed with a large density of cross-links and alternating connectivity of radical modules with unequal spin quantum numbers $(S)$, macrocyclic $S=2$ and, cross-linking $S=1 / 2$ modules, which permits large net $S$ values for either ferromagnetic or antiferromagnetic exchange couplings between the modules. In the highly cross-linked polymer, an effective magnetic moment corresponding to an average $S$ of about 5000 and slow reorientation of the magnetization by a small magnetic field (less than or equal to 1 oersted) below a temperature of about 10 kelvin are found. Qualitatively, this magnetic behavior is comparable to that of insulating spin glasses and blocked superparamagnets.
\end{abstract}

Recent investigations into the properties of organic magnets have generally concentrated on materials, such as crystalline solids of small molecule radicals or charge transfer salts, in which the exchange interaction involves s- and p-orbitals (1-5). An alternative approach to organic magnets may be based on $\pi$-conjugated polymers, as envisioned by Mataga in 1968 (6). Because exchange interactions between electron spin through the $\pi$-conjugated system can be made relatively strong, compared to the through-space interactions in molecular solids of organic radicals, this macromolecular approach has a potential for obtaining interesting magnetic properties at relatively high temperatures, even room temperature (7). Although significant progress has been made in the prepara-

Department of Chemistry, University of Nebraska, Lincoln, NE 68588-0304, USA.

*To whom correspondence should be addressed. Email: arajca1@unl.edu tion of $\pi$-conjugated oligomers and polymers with large values of spin quantum number $S$, organic polymer magnets have remained elusive $(8-15)$. We report the observation of magnetic properties comparable to that of insulating spin glasses and blocked superparamagnets in an organic $\pi$-conjugated polymer.

Our magnetic polymer, polymer $\mathbf{1}$, is designed with a large density of cross-links and alternating connectivity of radical modules with unequal spin quantum numbers, i.e., macrocyclic $S=2$ and cross-linking $S=1 / 2$ modules (13). This connectivity permits large net $S$ values for either ferromagnetic or antiferromagnetic exchange couplings between the modules (Fig. 1A). The cross-linking and connectivity is set in the synthesis of network polyether 2, precursor to polymer 1, based upon Pd-catalyzed Negishi coupling of two tetrafunctionalized macrocyclic monomers $\mathbf{3}$ and 4 (Fig. 1B) $(13,16)$.

Representative data for condensations
Fig. 1. (A) Polymer 1 with ferromagnetic or antiferromagnetic coupling between the macrocyclic $S=2$ and cross-linking $S=1 / 2$ modules. (B) Synthesis of polyether $\mathbf{2}$ and polymer 1. Reagents: (a) $t$-BuLi, THF, $198 \mathrm{~K}(2$ hours), $253 \mathrm{~K}$ (15 $\mathrm{min})$; (b) $\mathrm{ZnCl}_{2}$, from $198 \mathrm{~K}$ to ambient temperature; (c) $\mathrm{Pd}\left(\mathrm{PPh}_{3}\right)_{4}$ (3 mol \% per CC bond), THF, $373 \mathrm{~K}$ (10 min to 5 hours); (d) $\mathrm{Na} / \mathrm{K}$, 15-crown-5, THF-d ${ }_{8}, 283$ $\mathrm{K}$ (several days); and (e) $\mathrm{I}_{2}, 167$ to $170 \mathrm{~K}$.
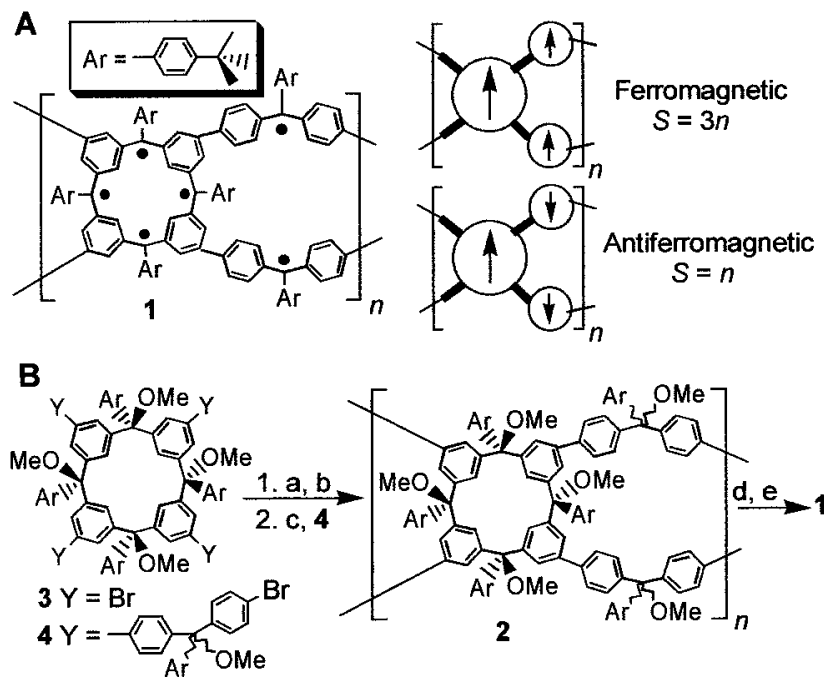

leading to organic insoluble polyether $\mathbf{2}$ are shown in Table $1(17,18)$. Dissolved metal (Na/K/15-crown-5) reduction of polyether 2 , as a gel with perdeuterated tetrahydrofuran $\left(\right.$ THF- $\left.\mathrm{d}_{8}\right)$, followed by the iodine oxidation of the corresponding carbopolyanion, gave polymer 1 (Fig. 1 and Table 1) (19).

The samples of polymer 1 obtained from polyethers $\mathbf{2}$ after long polymerization times (Table 1, run 3) have the most interesting magnetic behavior. Magnetic data of the thermally decomposed polymer 1 (essentially diamagnetic) and metal analyses both preclude any significant interference from magnetic metals on the reported magnetic behavior for polymer 1 (19). The magnetic field ( $H=0$ to $50 \mathrm{kOe})$ dependence of magnetization $(M)$, measured at several temperatures ( $T=1.8$ to $20 \mathrm{~K}$ ), shows an extraordinarily fast rise at low $H$; however, complete saturation is not attained even at $H=50 \mathrm{kOe}$ (Fig. 2A) (20). Numerical fits of the $M$ versus $H / T$ data $(T=1.8,2.5,3.5 \mathrm{~K})$ to a linear combination of one Langevin and three Brillouin functions give average spin quantum numbers $(S)$ and magnetizations at saturation $\left(M_{\text {sat }}\right)$ that are temperature-dependent (19, 21). Again, because ideal paramagnet (Curielike) behavior is not found, the maximum value of average $S \approx 5000$ at $3.5 \mathrm{~K}$, obtained
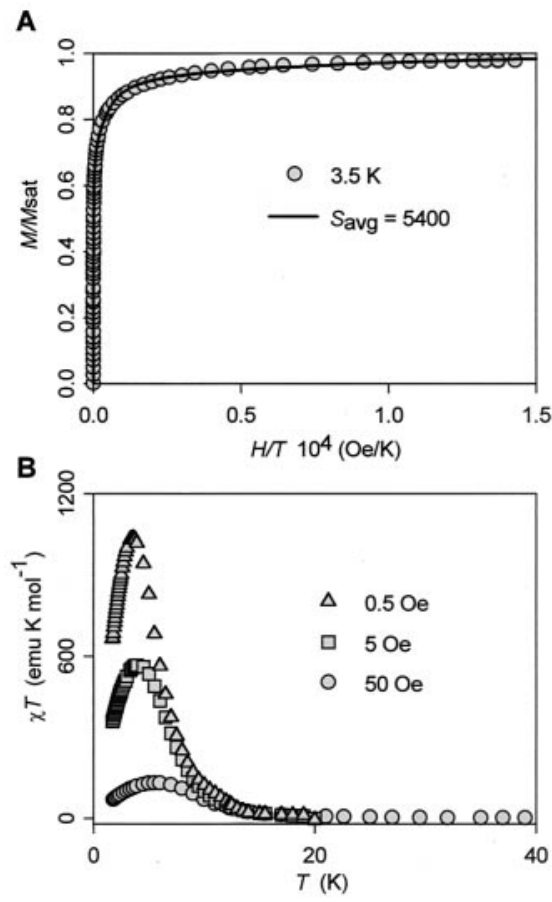

Fig. 2. (A) Magnetic field $(H)$ dependence of the magnetization $(M)$ of polymer 1 at $T=3.5 \mathrm{~K}$, plotted as $M / M_{\text {sat }}$ versus $H / T$, where $M_{\text {sat }}=$ $0.54 \mu_{B}$ is $M$ at saturation. The solid line corresponds to the least-squares fit using linear combination of Langevin and Brillouin functions corresponding to average $S=5400$ (19). (B) Plot of $\chi^{T}$ versus $T$ (dc susceptibility, $\chi=$ $M / H)$. 


\section{R E P O R T S}

from the $M$ versus $H / T$ plot, provides only a very approximate estimate for an effective ferro- or ferrimagnetic correlation of $10^{4}$ electron spin. Values of $M_{\mathrm{sat}}=0.5$ to $0.6 \mu_{\mathrm{B}}$ and their increase with increasing temperature are both compatible with the presence of weak antiferromagnetic and ferromagnetic interactions between the $S=2$ and $S=1 / 2$ modules in polymer 1 (Fig. 1).

The plots of $\chi T$ versus $T$ (dc susceptibility, $\chi=M / H$ ) show a rapid rise below about $10 \mathrm{~K}$. The values of $\chi T$ are highly fielddependent, as expected for very large magnetic moments (or values of $S$ ). For the smallest applied field, $H \approx 0.5 \mathrm{Oe}, \chi T$ reaches maximum of about 1000 electromagnetic units (emu) $\mathrm{K} \mathrm{mol}^{-1}$ at $3.6 \mathrm{~K}$ (Fig. 2B). Very approximately, this corresponds to an effective magnetic moment $\mu_{\mathrm{eff}} \approx 8000 \mu_{\mathrm{B}}$ or an average $S \approx 4000$, comparable to that found in the $M$ versus $H / T$ plots. These values may be viewed as lower bound estimates, because polymer 1 does not follow the Curie law and quantitative conversion of polyether $\mathbf{2}$ to polymer $\mathbf{1}$ is assumed.

More detailed studies of the temperature dependence of $M$ at low applied magnetic fields ( $H=0.5$ to $1.0 \mathrm{Oe}$ ) reveal that the zero field-cooled (ZFC) and the field-cooled (FC) magnetizations diverge below $\sim 10 \mathrm{~K}$, indicating the slow relaxation (blocking) of the magnetization (Fig. 3). This behavior is highly sensitive to the applied magnetic fields $(H)$; in larger magnetic fields, e.g., $H=5 \mathrm{Oe}$, the difference between the ZFC and FC magnetizations is undetectable. Even at a relatively low magnetic field, $H=5 \mathrm{Oe}$, the large values of $\mu_{\mathrm{eff}} \approx 8000$ give $\mu_{\mathrm{eff}} H / k_{\mathrm{B}} \approx 2.7$

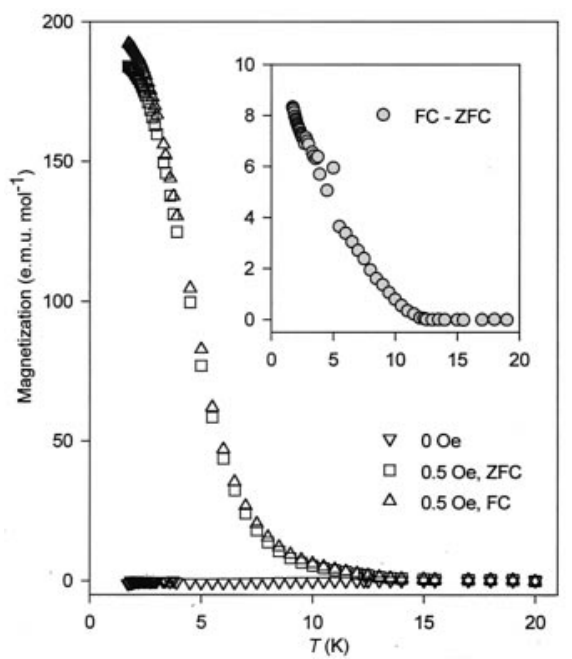

Fig. 3. Temperature dependence of the magnetization of polymer 1 measured by increasing the temperature in the indicated field. For $H=0.5$ Oe measurements, the sample was cooled either in the zero-field (ZFC) or in the 0.5 Oe field $(F C)$ prior to the measurement. (Inset) Difference between the FC and ZFC magnetization.
$\mathrm{K}$, which is within the range of blocking temperatures.

The relaxation of the magnetization for polymer $\mathbf{1}$ at low temperatures is best observed in the temperature and frequency dependence of the ac susceptibility. The temperature dependence of $\chi^{\prime}$ (in-phase component) shows a steep rise around $10 \mathrm{~K}$, with a broad peak in the 1.9 to $3 \mathrm{~K}$ region (Fig. 4A) (19). The peak maximum in $\chi^{\prime}$ at $T_{\mathrm{m}}{ }^{\prime}$ shifts to lower temperatures, and it gains intensity with decreasing frequency. The out-of-phase component, $\chi^{\prime \prime}$, is detectable at about $10 \mathrm{~K}$ and shows a frequency-dependent maximum in the 1.8 to $2.0 \mathrm{~K}$ region (Fig. 4B). The peak maximum in $\chi^{\prime \prime}$ at $T_{\mathrm{m}}$ " shifts to lower temperatures and becomes less intense with decreasing frequency; at lower frequencies, e.g., $v_{\mathrm{ac}}<250 \mathrm{~Hz}$, only continuous increase of $\chi^{\prime \prime}$ is found down to $1.7 \mathrm{~K}$, the lower limit for the temperature control in our supercon-

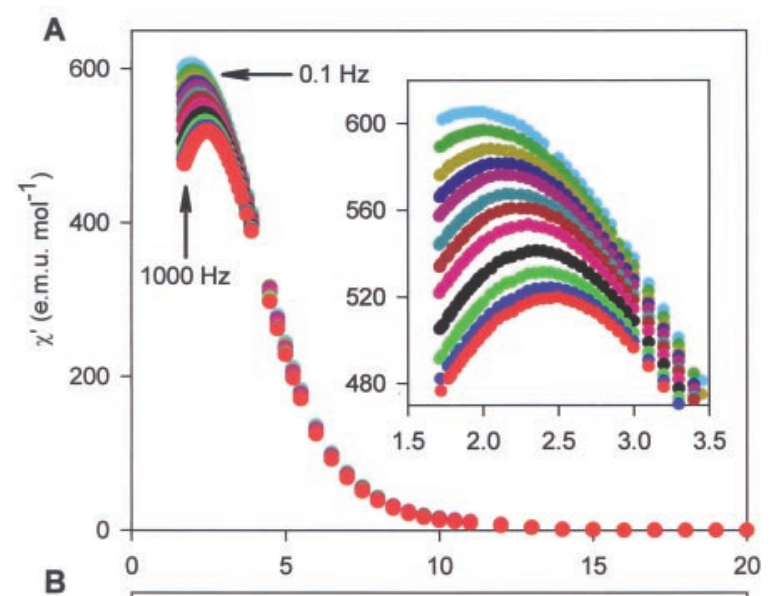

Fig. 4. Temperature dependence of the ac susceptibility $\chi^{\prime}$ (A) and $\chi^{\prime \prime}(B)$ of polymer 1 measured in the zero applied field. The ac driving field is 0.1 Oe and the frequencies are 1000,800 , $500,250,100,50,25,10,5,2$, 0.5 , and $0.1 \mathrm{~Hz}$. [Diameter of the symbols for $\chi^{\prime \prime}$ in the inset of (B) represents approximately one standard deviation for three measurements.] 


\section{R E P O R T S}

time of the magnetization. With the very few $T_{\mathrm{m}}$ " data points and narrow frequency range, the plot of $\ln (\tau)$ versus $1 / T$ gives a straight line $(R=0.996)$ with an order of magnitude estimates for the barrier $E_{\mathrm{A}} / k_{\mathrm{B}}=15 \mathrm{~K}$ and the microscopic limiting relaxation time $\tau_{0}=$ $9 \times 10^{-8} \mathrm{~s}$. Both $E_{\mathrm{A}} / k_{\mathrm{B}}$ and $\tau_{0}$ are comparable to those found in slowly relaxing molecular cluster-based superparamagnets (e.g., in $\mathrm{Fe}_{8}, E_{\mathrm{A}} / k_{\mathrm{B}}=22.2 \mathrm{~K}$ and $\tau_{0}=1.9 \times 10^{-7}$ s) (25).

Analogous to ZFC/FC magnetizations, both $\chi^{\prime}$ and $\chi^{\prime \prime}$, as measured at the frequency of $1000 \mathrm{~Hz}$ and the ac driving field of $0.1 \mathrm{Oe}$, are highly sensitive to the applied $H$. For $H \approx$ 0.5 and $1 \mathrm{Oe}, T_{\mathrm{m}}{ }^{\prime}$ shifts from $2.50 \mathrm{~K}$ (at zero-field) to 2.10 and $1.75 \mathrm{~K}$, respectively; the intensity is significantly lowered (at $1 \mathrm{Oe}$, $60 \%$ of the zero-field value). The $T_{\mathrm{m}}{ }^{\prime \prime}$ shifts to $\leq 1.7 \mathrm{~K}$ even at $0.5 \mathrm{Oe}$ and the intensity decrease of $\chi^{\prime \prime}$ is even more pronounced than for $\chi^{\prime}$ (at $1 \mathrm{Oe}, 25 \%$ of the zero-field value at $1.7 \mathrm{~K})$.

Polyethers 2 obtained after short polymerization times (Table 1 , runs 1 and 2) give polymers 1 with relatively lower values of average $S=600$ to 1500 (seven samples). No peaks in ac susceptibility are detected; however, a small and frequency-dependent $\chi^{\prime \prime}<1$ emu $\mathrm{mol}^{-1}$ is observed at low temperatures, suggesting an onset of magnetic blocking.

In conclusion, our experimental data show that organic polymer magnets can be prepared. In polymer 1, both blocking of magnetization and very large magnetic moments are found below a temperature of about $10 \mathrm{~K}$. Overall, the magnetic behavior falls between insulating spin glasses and blocked superparamagnets, but closer to spin glasses.

\section{References and Notes}

1. M. Kinoshita, in Handbook of Organic Conductive Molecules and Polymers, H. S. Nalva, Ed. (Wiley, New York, 1997), vol. 1, chap. 15.

2. P.-M. Allemand et al., Science 253, 301 (1991).

3. B. Narymbetov et al., Nature 407, 883 (2000).

4. M. Mito et al., Polyhedron 20, 1509 (2001).

5. W. Fujita, K. Awaga, Science 286, 261 (1999).

6. N. Mataga, Theor. Chim. Acta 10, 372 (1968).

7. A. Rajca, Chem. Rev. 10, 871 (1994).

8. N. Nakamura, K. Inoue, H. Iwamura, Angew. Chem. Int. Ed. 32, 872 (1993).

9. A. Rajca, J. Wongsriratanakul, S. Rajca, R. Cerny, Angew. Chem. Int. Ed. 37, 1229 (1998)

10. R. J. Bushby, D. R. McGill, K. M. Ng, N. Taylor, J. Mater. Chem. 7, 2343 (1997)

11. K. K. Anderson, D. A. Dougherty, Adv. Mater. 10, 688 (1998).

12. H. Nishide, M. Miyasaka, E. Tsuchida, Angew. Chem. Int. Ed. 37, 2400 (1998).

13. A. Rajca, S. Rajca, J. Wongsriratanakul, J. Am. Chem. Soc. 121, 6308 (1999).

14. P. M. Lahti, Ed., Magnetic Properties of Organic Materials (Dekker, New York, 1999).

15. I. Itoh, M. Kinoshita, Eds., Molecular Magnetism, New Magnetic Materials (Gordon \& Breach, Amsterdam, 2000).

16. P. J. Flory, Principles of Polymer Chemistry (Cornell Univ. Press, Ithaca, NY, 1953).

17. The ${ }^{1} \mathrm{H}$ NMR $(500 \mathrm{MHz}, 348 \mathrm{~K})$ spectra of polyether 2 gelled with benzene- $d_{6}$ are identical for different polymerization times. The three broad peaks $(7.5,3$, and $1 \mathrm{ppm}$ ) have the chemical shifts as expected for the aromatic, methoxy, and tert-butyl protons of polyether 2. Analogously, ${ }^{13} \mathrm{C}$ NMR $(125 \mathrm{MHz})$ spectrum has four broad resonances at $88,53,34$, and 32 ppm, corresponding to the triarylmethyl, methoxy, quaternary tert-butyl, and methyl tert-butyl carbons; the aromatic region is obstructed by the benzene- $d_{6}$ peak.

18. The minor, organic soluble polyether $2\left(M_{w}=3-\right.$ $\left.5 \times 10^{5} \mathrm{Da}\right)$ gave paramagnetic polymers with an average $S \geq 40$ (13). These values of $M_{w}$ may be considered as the lower ends of the molecular weight distributions for entries in Table 1.

19. Experimental procedures involving generation of polymer 1, magnetic measurements, numerical fitting of magnetic data, determination of magnetic impurities are available on Science Online (www. sciencemag.org/cgi/content/full/294/5546/1503/ DC1).
20. All magnetic measurements were carried out using Quantum Design DC/AC SQUID MPMS5S magnetometer.

21. The values of average $S$ [or $S(S+1)$ ] follow qualitatively the $\chi T$ versus $T$ plot (Fig. 2B).

22. R. Sessoli, D. Gatteschi, A. Caneschi, M. A. Novak, Nature 365, 141 (1993).

23. J. A. Mydosh, Spin Glasses, an Experimental Introduction (Taylor \& Francis, London, 1993).

24. T. Jonsson, P. Nordblad, P. Svedlindh, Phys. Rev. B 57, 497 (1998).

25. A. L. Barra, P. Debrunner, D. Gatteschi, Ch. E. Schulz, R. Sessoli, Europhys. Lett. 35, 133 (1996).

26. Financial support was provided by the NSF. We thank M. Carlson (University of Nebraska, Lincoln) for the help with ICP analyses and S.-H. Liou (UNL, Department of Physics) for reading the manuscript.

17 August 2001; accepted 15 October 2001 